\title{
Modeling of the Be Stars
}

\author{
K. Šejnová ${ }^{1,2}$ V. Votruba ${ }^{1,2}$ and P. Koubskýy ${ }^{1}$ \\ ${ }^{1}$ Astronomical Institute AV ČR v.v.i, Ondřejov, Czech Republic \\ ${ }^{2}$ Masaryk University, Faculty of Science, Brno, Czech Republic
}

\begin{abstract}
The Be stars are still a big unknown in respect to the origin and geometry of the circumstellar disk around the star. Program shellspec is designed to solve the simple radiative transfer along the line of sight in three-dimensional moving media. Our goal was to develop an effective method to search in parameter space, which can allow us to find a good estimate of the physical parameters of the disk. We also present here our results for Be star 60 Cyg using the modified code.
\end{abstract}

Keywords. Be stars, shellspec, genetic algorithms

\section{Introduction}

Program shellspec is designed by J. Budaj (Budaj \& Richards 2004). Optional (non)transparent objects such as spot, disk, shell, etc. may be defined in 3D and their composite synthetic spectrum calculated. The main input file is called shellspec.in. We can set input parameters of an object here. In the current version of the code, the parameters have to be set manually, which is not an effective method, so we decided to make the program more automatic.

\section{Modification of shellspec}

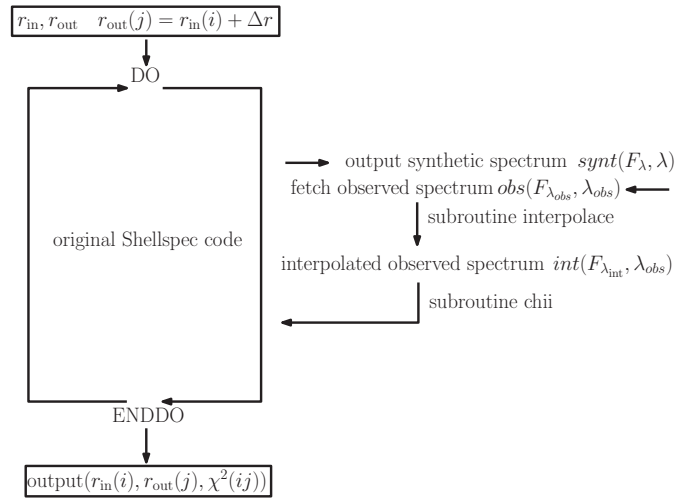

Figure 1. Procedure to modify shellspec program

The first part of the modification includes a grid method that systematically searches through the field of control parameters. Then the calculation is repeated, and as a result we get the synthetic spectrum, which we compare with the observed one. On the basis of the known observed spectrum, we find the value of $\chi^{2}$ from the match. The last part of the calculation consists of choosing the best combination of control parameters that describes the observed spectrum in the best way when the chosen criterium is the $\chi^{2}$ value. 


\section{Results for 60 Cyg}

We used a modified version of the shellspec code to study 60 Cyg. The results are shown in Table 1 and Fig. 2.

Table 1. Final parameters of disk of 60 Cyg star

\begin{tabular}{c|cccccc} 
& $i$ & $R_{\text {in }}\left[\mathrm{R}_{\odot}\right]$ & $R_{\text {out }}\left[\mathrm{R}_{\odot}\right]$ & $T[\mathrm{~K}]$ & $\rho\left[\times 10^{-13} \mathrm{~g} \mathrm{~cm}^{-3}\right]$ & $v_{\text {trb }}\left[\mathrm{km} \mathrm{s}^{-1}\right]$ \\
\hline P1 & 30 & 5,202 & 12,681 & 13000 & 9 & 90 \\
P2 & 30 & 6,979 & 7,748 & 13000 & 1 & 60 \\
P23 & 30 & 5,202 & 6,742 & 14000 & 1 & 80 \\
P233 & 30 & 5,455 & 5,982 & 14600 & 1 & 70 \\
P3 & 30 & 5,202 & 5,532 & 14500 & 2 & 80
\end{tabular}
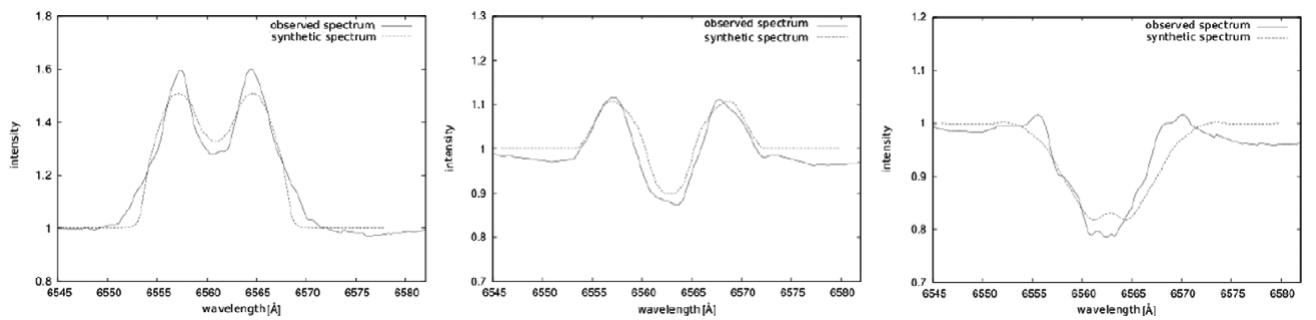

Figure 2. Observed and synthetic spectrum for three different $\mathrm{H} \alpha$ profiles of $60 \mathrm{Cyg}$

\section{PIKAIA subroutine}

The grid method is not one of the most effective methods due to its computational heftiness. A genetic algorithm can be used to improve the computational efficiency because it would allow us to increase the number of control parameters. Genetic Algorithms are search and optimization procedures that are motivated by the principles of natural genetics and natural selection. PIKAIA is a general purpose optimization subroutine based on a genetic algorithm (Charbonneau \& Knapp 1995).

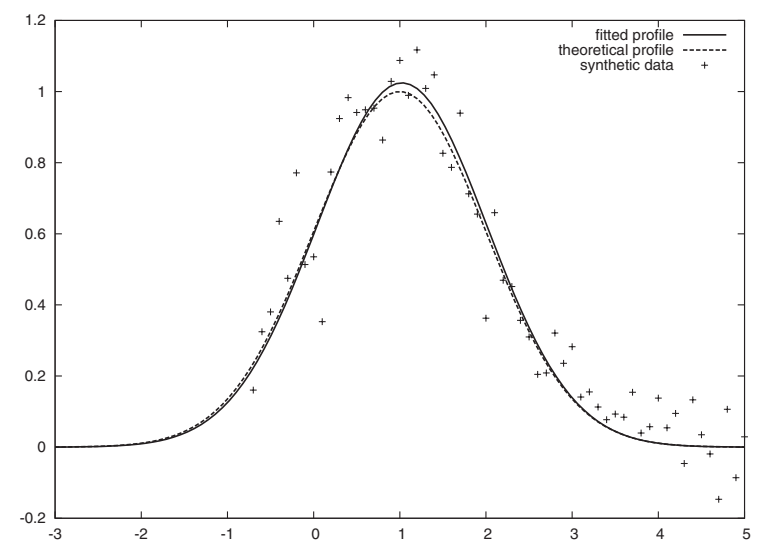

Figure 3. Test example: gaussian curve fit where theoretical curve has shape $f(x)=e^{-\frac{(x-1)^{2}}{2}}$

\section{References}

Šejnová, K. 2010, Shell modeling of the Be stars, Diploma Thesis, MU Brno

Budaj, J. \& Richards, M. T. 2004, A description of the Shellspec code, Contrib. Astron. Obs. Skalnate Pleso 34, 1-30

Koubský, P., et al. 2000, ASP-CS, 214, 280

Charbonneau, P. \& Knapp, B. 1995, A User's Guide To PIKAIA 1.0, Vol. NCAR/TN-418+IA 